\title{
Nasogastric and intravenous infusions of (+)-4-propyl-9-hydroxynaphthoxazine (PHNO) in Parkinson's disease
}

\author{
R J Coleman, N P Quinn, M Traub, C D Marsden
}

\begin{abstract}
(+)-4-propyl-9-hydroxynaphthoxazine (PHNO) is a new dopamine agonist which is capable of producing a sustained response in parkinsonian patients with "onoff' fluctuations when given as a continuous infusion either nasogastrically or intravenously. These data suggest that a sustained release formulation of PHNO may provide a significant, new treatment for patients with "on-off' fluctuations.
\end{abstract}

In patients with Parkinson's disease, the management of "on-off" fluctuations still represents a major clinical challenge. Fluctuations can be improved and sometimes abolished by an intravenous infusion of levodopa. ${ }^{3}$ This technique is impractical for routine clinical use, but nevertheless represents the best model available for an ideal therapy. Various attempts have been made to adapt the concept of continuous levodopa therapy for use in patients with "on-off" fluctuations. One approach involves slow-release oral levodopa tablets; ${ }^{4-6}$ another makes use of dopamine agonist drugs (for example, lisuride and apomorphine) which can be delivered by continuous subcutaneous infusion using a portable battery-operated pump. ${ }^{78}$

(+)-4-propyl-9-hydroxynaphthoxazine (PHNO) is a new, highly potent dopamine agonist, selectively active at the $\mathrm{D} 2$ receptor site, which has been shown to possess antiparkinsonian activity. ${ }^{9-11}$ It is possible that such a drug might be used in the treatment of "onoff " fluctuations if it could be delivered in a continuous manner.

This study describes our experience with PHNO given via nasogastric and intravenous infusion. Continuous nasogastric infusions were employed to mimic the effect of a slowrelease tablet. Intravenous infusions were used to simulate direct systemic administration that might be achieved by a subcutaneous infusion or a transdermal patch.

\section{Patients and methods}

Eight patients with idiopathic Parkinson's disease took part in two studies having given written informed consent; their clinical details are shown in the table. Six patients received nasogastric infusions and five had intravenous infusions; three patients took part in both studies. They were all taking regular oral levodopa with a peripheral decarboxylase inhibitor, and all experienced "on-off" fluctuations in response to this treatment. During infusions all patients received oral domperidone ( $60 \mathrm{mg}$ daily).

No antiparkinsonian drugs were taken after midnight on each of the study days and so the patients were always "off" in the morning when the infusion was started. The response to each infusion was recorded at the bedside by regular assessment using an accurate "on-off" chart and the total length of time spent "on" was taken as a convenient measure of drug response. $^{12}$

PHNO was diluted in $0.9^{\circ}{ }_{0}$ saline and infused using a portable battery-operated pump. (Graseby Driver 16A) connected to either a fine bore nasogastric tube or a fore-arm intravenous cannula. The infusion rates used were between $0.5 \mathrm{mg} / \mathrm{hr}$ and $2.0 \mathrm{mg} / \mathrm{hr}$ during the nasogastric study and between $2 \mu \mathrm{g} / \mathrm{hr}$ and $32 \mu \mathrm{g} / \mathrm{hr}$ in the intravenous study. (Before each nasogastric infusion, an hour's dose of drug was given as a bolus.) If no response occurred during the first four hours of an infusion (that is, the patient did not turn "on"), the infusion was stopped and the usual levodopa therapy was restarted; a higher infusion rate was then used on a subsequent day. When a response was observed the infusion was continued for up to eight hours. A washout period of at least 16 hours was allowed between infusions.

Venous samples were drawn at regular intervals for subsequent measurement of PHNO levels. (During the intravenous study, samples were taken from the arm opposite the infusion site.) Measurement of PHNO plasma concentration was carried out using a radioimmunoassay, details of which will be published elsewhere. Antibodies were raised using 9-O-carboxypropyl-PHNO coupled to bovine serum albumin as the immunogen. The radioligand was 8-iodo(I-125)-PHNO. A double antibody radioimmunoassay was established with a $1 \mathrm{ml}$ total volume using antisera at a final dilution of up to 5 million, and a sensitivity of $0.3 \mathrm{pg}$ per tube. Fifty per cent inhibition of ligand binding occurred at about $3 \mathrm{pg}$ per tube. Biological samples were extracted at $\mathrm{pH} 9.2$ with methyl t-butyl ether and the drug was back-extracted into $0.01 \mathrm{n}$ hydrochloric acid. Up to $0.2 \mathrm{ml}$ of plasma was assayed yielding a sensitivity of about $2 \mathrm{pg}$ PHNO per ml plasma. Recoveries were greater than $90^{\circ}{ }_{0}$ and independent of concentration. 
Table Details of patients

\begin{tabular}{|c|c|c|c|c|c|c|c|}
\hline \multirow[b]{2}{*}{ Patient } & \multirow[b]{2}{*}{ Sex } & \multirow[b]{2}{*}{ Age } & \multirow[b]{2}{*}{ Study } & \multicolumn{3}{|c|}{ Duration in years of: } & \multirow[b]{2}{*}{$\begin{array}{l}\text { Levodopa dose } \\
(\text { mg })_{\text {day })^{\star}}\end{array}$} \\
\hline & & & & $P D$ & $\begin{array}{l}\text { Levodopa } \\
\text { therapy }\end{array}$ & On-off & \\
\hline $\begin{array}{l}1 \mathrm{RE} \\
2 \mathrm{LE} \\
3 \mathrm{HG} \\
4 \mathrm{GL} \\
5 \mathrm{DC} \\
6 \mathrm{WR} \\
7 \mathrm{GG} \\
8 \mathrm{RC}\end{array}$ & $\begin{array}{l}M \\
F \\
M \\
M \\
M \\
M \\
M \\
M\end{array}$ & $\begin{array}{l}49 \\
52 \\
64 \\
44 \\
53 \\
38 \\
44 \\
56\end{array}$ & $\begin{array}{l}\text { NG } \\
\text { NG } \\
\text { NG } \\
\text { NG/IV } \\
\text { NG/IV } \\
\text { NG/IV } \\
\text { IV } \\
\text { IV }\end{array}$ & $\begin{array}{r}8 \\
17 \\
8 \\
11 \\
21 \\
6 \\
8 \\
19\end{array}$ & $\begin{array}{r}7 \\
13 \\
4 \\
11 \\
16 \\
6 \\
5 \\
18\end{array}$ & $\begin{array}{r}5 \\
10 \\
1 \\
8 \\
8 \\
3 \\
4 \\
6\end{array}$ & $\begin{array}{r}700 \\
1100 \\
800 \\
950 \\
2100 \\
800 \\
1300 \\
1375\end{array}$ \\
\hline
\end{tabular}

$\star=$ with peripheral decarboxylase inhibitor.

\section{Results}

(i) Nasogastric infusions

All six patients switched "on" at some stage during one of the nasogastric infusions. The response was similar to that achieved with levodopa and the usual range of "on-off" phenomena were observed, including peakdose and diphasic dyskinesias. When the lowest infusion rate was used, the patients showed no consistent response. With intermediate doses (that is, suboptimal infusion rates) the patients turned "on" and then "off" again, sometimes many times, despite a constant infusion rate. As the infusion rate was increased, the patients experienced longer and more sustained "on" periods on successive days. The best response observed was a continuous "on" period lasting for six and a half hours of an eight hour infusion. When the patient was still "on" at the end of an infusion (which happened on a total of five occasions) the post-infusion "on" period continued for between 0.75 and 2.3 hours.

During each nasogastric infusion there was an apparent relationship between the proportion of the infusion time spent "on" and the rate of PHNO infusion (fig 1). The mean plasma levels of PHNO (measured in picograms per millilitre) at 0,4 and 8 hours were higher with increasing infusion rate (fig 2). Insufficient samples were collected either to correlate plasma levels with clinical response or to permit detailed pharmacokinetic analysis.

\section{(ii) Intravenous infusions}

All five patients switched "on" in response to PHNO during one of the intravenous infusions. Four of the five patients showed their first clinical response while receiving PHNO at a rate of $16 \mu \mathrm{g} / \mathrm{hr}$, and the fifth responded to $32 \mu \mathrm{g} / \mathrm{hr}$. Both for individual patients and the group as a whole, the duration of the "on" response increased in relation to the

Figure 1 Clinical response to increasing rates of nasogastric infusion of PHNO

The length of time for which each patient was "on", expressed as a percentage of the total infusion time, is used as a measure of clinical response.

$10=$ individual patients; $\mathbf{O}=$ mean).

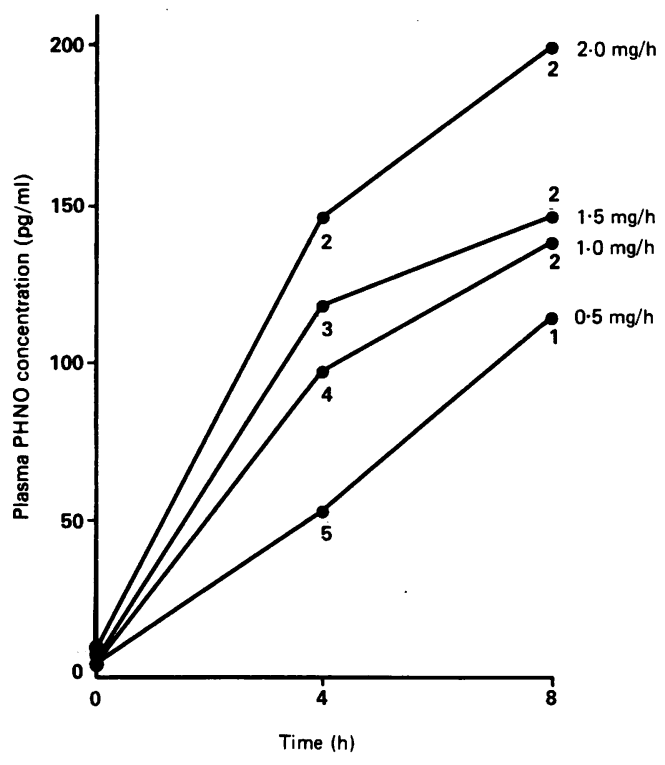

Figure 2 Mean plasma PHNO concentrations with increasing nasogastric infusion rate

Beside each point is shown the number of observations contributing to that mean.

infusion rate (fig 3 ). The three patients able to tolerate an infusion rate of $32 \mu \mathrm{g} / \mathrm{hr}$ enjoyed a sustained antiparkinsonian response, staying "on" for between $85^{\circ}{ }_{0}$ and $93^{\circ}{ }_{0}$ of the duration of the infusion.

The PHNO concentration rose with increasing infusion rate. The results for patients 4 and 6 are shown as typical examples (fig 4 ). The levels rose rapidly from the start of each infusion and in most cases began to plateau after 3 to 4 hours. However in individual patients the PHNO levels continued to fluctuate despite continuous drug delivery. There were insufficient data for accurate calculation of the steady state plasma PHNO concentration for each infusion, but this was estimated by averaging the results from hour 3 to the end of the infusion. The steady state PHNO concentrations estimated in this way bore a clear relationship to the rate of $\mathrm{PHNO}$ infusion (fig 5). After the end of each infusion PHNO levels fell rapidly. The samples taken 1,2 and 4 hours after the end of the infusion were calculated as a percentage of the preceding estimated steady state plasma level (fig 6).

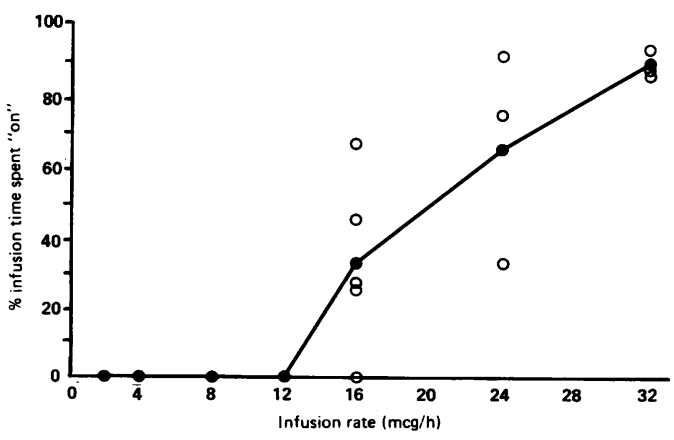

Figure 3 Clinical response to increasing rates of intravenous infusion of $P H N O$

The length of time for which each patient was "on", expressed as a percentage of the total infusion time, is used as a measure of clinical response.

$(\mathrm{O}=$ individual patients; $\mathrm{O}=$ mean $)$. 
Figure 4 Plasma PHNO concentrations during and after intravenous infusions in patients 4 and 6

Solid lines $=$ during infusion; broken lines = after infusion discontinued. Rates of infusion: $\bigcirc$,

$\square, \boldsymbol{\square}=8 \mu \mathrm{g} / \mathrm{hr}$,

$16 \mu g / h r, 24 \mu g / h r$

$32 \mu \mathrm{g} / \mathrm{hr}$ respectively.
Figure 5 Steady rate PHNO concentrations in response to increasing rates of intravenous infusion of PHNO

For each patient, a steady state PHNO concentration was estimated by

averaging the individual

PHNO concentrations

from hour 3 to the end of

the infusion. ( $, \square, 0$,

$\square, \boldsymbol{\Delta}=$ Patients 4, 5, 6 ,

7,8 respectively.)

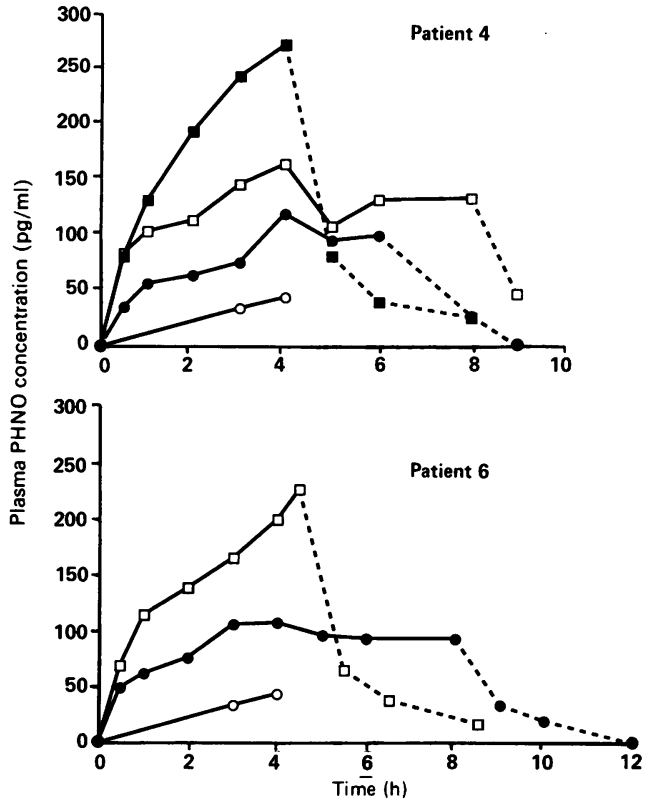

These data suggest that the plasma half-life of PHNO is approximately one hour.

There was no consistent plasma PHNO concentration which always produced a clinical effect. Levels below $60 \mathrm{pg} / \mathrm{ml}$ were very rarely effective whereas levels above $200 \mathrm{pg} / \mathrm{ml}$ were always associated with an "on" response. Between these limits, higher drug concentrations were associated with an increased probability of the patient being "on"; with concentrations in the region of $100 \mathrm{pg} / \mathrm{ml}$, there was about a $50 \%$ chance of the patient being "on".

\section{(iii) Adverse effects}

All patients experienced dyskinesias in response to PHNO which were usually choreiform in nature and could not be distinguished in appearance from those usually associated with levodopa therapy. Dyskinesias only occurred during "on" periods and their presence was not related to PHNO dose or plasma concentration. In this group of severely affected patients, it appeared impossible to achieve an "on" response without some dyskinesias.

Apart from dyskinesias, the commonest side effect was drowsiness which occurred in three out of six patients during the nasogastric study and in four out of five patients in the intravenous study. The severity of the drowsiness

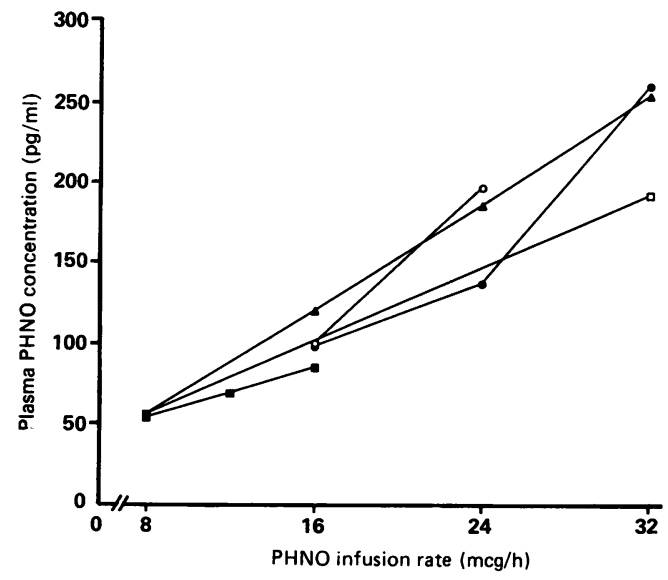

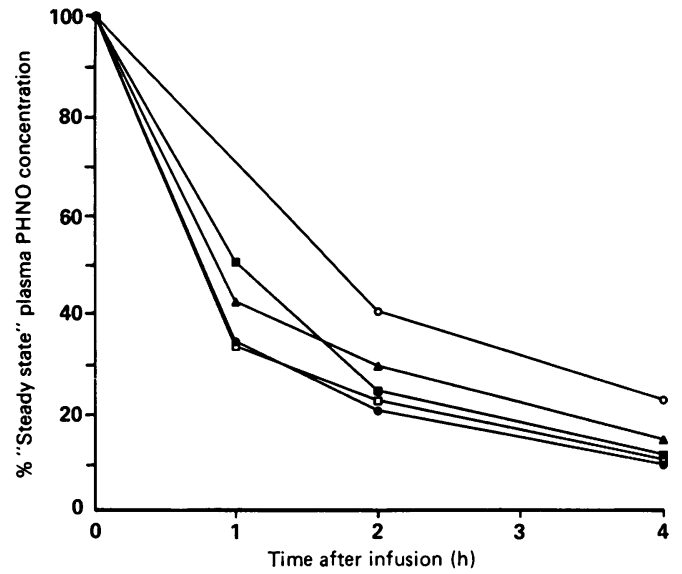

Figure 6 The fall in PHNO concentration following the termination of intravenous infusions of PHNO.

At 1,2 and 4 hours after the termination of an intravenous infusion, the PHNO concentration is expressed as a percentage of the preceeding steady state concentration. (Each line represents the mean result for one patient: $\square, \Delta, \bigcirc, \bigcirc, \square=$ Patients $4,5,6,7,8$ respectively.)

appeared to be dose related. In one patient drowsiness was associated with confusion and visual distortions, which cleared several hours after stopping the infusion. One patient vomited and two experienced nausea despite domperidone.

\section{Discussion}

All patients switched "on" in response to PHNO, as predicted from its pharmacological properties and as reported in previous clinical studies using single oral doses. ${ }^{9-11}$ Higher rates of drug delivery gave rise to larger percentages of the infusion time spent "on". At best, continuous delivery of PHNO, using either the nasogastric or the intravenous route, was able to produce a stable response over many hours without levodopa.

Plasma PHNO concentrations taken after each intravenous infusion suggest that the halflife of PHNO was approximately one hour. This is consistent with the observation that an apparent steady state was reached approximately four hours after the intravenous infusion was started. This estimated half-life of PHNO is also consistent with the duration of clinical effect after a nasogastric infusion was discontinued and with the previously reported duration of action of single oral doses in a similar group of patients. ${ }^{11}$

The doses of nasogastric PHNO required to achieve therapeutic blood levels were about sixty-fold higher than those required for the intravenous route, probably due to a high degree of first pass metabolism (unpublished data).

The side effects experienced with PHNO were those that might be expected of a potent dopamine agonist, namely drowsiness, nausea and vomiting in addition to the usual range of dyskinesias. These appeared to be dose related and all resolved completely when PHNO was discontinued.

The results of our study support the potential use of PHNO as an antiparkinsonian agent 
and show that PHNO, given by continuous infusion, may be useful in stabilising patients with "on-off" fluctuations. Although nasogastric and intravenous infusions do not represent a practical method for the day to day management of patients, it is hoped that the techniques may be adapted for more general use. A nasogastric infusion mimics the effect of a slow-release oral tablet ${ }^{13}$ and a parenteral infusion is similar to what might be expected from transcutaneous delivery using a skin patch. ${ }^{1+15}$

At present, a subcutaneous infusion of the dopamine agonist apomorphine in conjunction with intermittent oral levodopa seems to provide the best control for those patients with the most severe oscillations. ${ }^{8}$ Continuous oral or transdermal PHNO therapy might achieve a similar beneficial effect, avoiding the need for needles and pumps. It is not known whether the D2 selectivity of PHNO will have any influence on its effectiveness or its clinical uses. Apomorphine is a potent agonist at both D1 and D2 receptors and as such may be said to more closely reproduce the pharmacological properties of dopamine. Future comparison of apomorphine and PHNO may provide clues to the different roles of $\mathrm{D} 1$ and $\mathrm{D} 2$ receptors in Parkinson's disease.

Further study is warranted to investigate the various possible routes of PHNO administration and the different ways in which PHNO and levodopa therapies might be combined. The future role of this drug remains to be determined.

We thank Dr M Hichens of Merck, Sharp and Dohme Research Laboratories, West Point, Pennsylvania, United States, for carrying out the PHNO assay and for providing details of the method used.

1 Hardie RJ, Lees AJ, Stern G. On-off fluctuations in Parkinson's disease. Brain 1984;107:487-506.

2 Nutt JG, Woodward WR, Hammerstad JP, Carter JH, Anderson JL. The on-off phenomenon in Parkinson's disease. N Eng J Med 1984;310:483-8.

3 Quinn NP, Parkes JD, Marsden CD. Control of on-off phenomenon by continuous intravenous infusion of levodopa. Neurology 1984;34:1131-6.

4 Juncos FL, Fabbrini G, Mouradian MM, Serrarti C, Kask $A M$, Chase TN. Controlled release levodopa treatment of motor fluctuations in Parkinson's disease. J Neurol motor fluctuations in Parkinson's
Neurosurg Psychiatry 1987;50:194-8.

5 Cedarbaum JM, Breck L, Kutt H, McDowell FH. Controlled-release levodopa/carbidopa. I. Sinemet CR3 treatment of response fluctuations in Parkinson's disease. Neurology 1987;37:233-41.

6 Cedarbaum JM, Breck L, Kutt H, McDowell FH. Controlled-release levodopa/carbidopa. II. Sinemet CR4 treatment of response fluctuations in Parkinson's disease. Neurology 1987;37:1607-12.

7 Obeso JA, Luquin MR, Martinez-Lage JM. Lisuride infusion pump: a device for the treatment of motor infusion pump: a device for the treatment of motor

8 Stibe CMH, Lees AJ, Kempster PA, Stern GM. Subcutaneous apomorphine in parkinsonian on-off oscillations. Lancet 1988;i:403-6.

9 Martin GE, Williams M, Pettibone DJ, Yarbrough GG, Clineschmidt BV, Jones JH. Pharmacological profile of a novel potent directly-acting dopamineagonist, $(+)-4-$ propyl-9-hydroxynaphthoxazine((+)-PHNO). J Pharmacol Exp Ther 1986;230:569-6.

10 Stoessl AJ, Mak E, Calne DB. (+)-4-propyl-9-hydroxynaphthoxazine (PHNO), a new dopaminomimetic, in treatment of parkinsonism. Lancet 1985;ii:1330-1.

11 Grandas F, Quinn N, Critchley P, Rohan A, Marsden CD, Stahl SM. Antiparkinsonian activity of a single dose of PHNO. Movement Disorders 1987;2:47-51.

12 Coleman RJ, Temlett JA, Quinn NP, Stahl SM, Marsden CD. A strategy for measuring the therapeutic properties of $\mathrm{CD}$. A strategy for measuring the therapeutic properties of
new antiparkinsonian drugs, such as $(+)-\mathrm{PHNO}$, in new antiparkinsonian drugs, such as $(+)$-PHNO, in
patients with on-off fluctuations. Clin Neuropharmacol patients with
$1989 ; 12: 37-45$

13 Lieberman A, Chin L, Baumann G. MK458, a selective and potent D2 receptor agonist in advanced Parkinson's disease. Clin Neuropharmacol 1988;11:191-200.

14 Rupniak NMJ, Tye SJ, Jennings CA, et al. Antiparkinsonan efficacy of a novel transdermal delivery system for (+)-PHNO in MPTP-treated squirrel monkeys. Neurology 1989;39:329-35.

15 Coleman RJ, Lange KW, Quinn NP, et al. The antiparkinsonian actions and pharmacokinetics of transdermal $(+)$ 4-propyl-9-hydroxynapthoxazine (PHNO): preliminary results. Movement Disorders 1989;4:129-38. 